ANGELA MARIA MAZZANTI

\title{
GLI AVVENIMENTI STORICI COME RIVELAZIONE L'INCIPIT DELLA LEGATIO AD CAIUM DI FILONE DI ALESSANDRIA
}

\begin{abstract}
Summary: In the incipit of Legatio ad Caium of Philo of Alexandria anthropological and theological concepts are expressed which do not appear immediately relevant to the story: the narration of dramatic historical circumstances which the author personally lived through. The analysis of the specific contents reveals the connection betwen fundamental religious and cultural themes which are reoccurring themes in the various works of Philo. The introduction does not place ideology a priori but his considerations are evidence of a method of judgement of historical events.
\end{abstract}

Key words: Philo of Alexandria, introduction of Legatio ad Caium, theological and anthropological concepts

L'incipit della Legatio ad Caium di Filone di Alessandria enuncia una serie di concezioni la cui congruenza con la trattazione "storica"1 appare problematica per vari aspetti. Il passo è stato oggetto di varie interpretazioni ma può essere proposta un'ulteriore analisi che innanzitutto tenga conto del quadro in cui tale introduzione trova collocazione e non si sottragga, nel contempo, seppure tramite attestazioni paradigmatiche, alla cognizione dell'orizzonte complessivo del pensiero dell' esegeta.

${ }^{1}$ Si può parlare di opera storica? C. KRAUS (Filone Alessandrino e un'ora tragica della storia ebraica. Napoli 1967, 35-38) ritiene che ci sia un ritmo narrativo più drammatico che storico e precisa la presenza di un rapporto dinamico fra tematiche divergenti: «Considerando ormai nota l'impostazione filosofico-religiosa del pensiero filoniano e anticipando la nozione che l'autore non indaga sulle ragioni politico-sociali del pogrom, si presenta questa alternativa: o l'avvenimento storico è circoscritto al suo valore episodico e il commento concettuale inserito nella trattazione ha come fine l'inquadramento drammatico dell'episodio stesso; oppure, nel corso della narrazione, la materia storica diviene tramite al chiarimento di una tesi etico-religiosa, secondo la quale la storia dell'uomo - e non dell'ebreo soltanto - è regolata da una legge universale che lega il destino umano alla trascendenza di Dio». Le due prospettive, termina la studiosa, nella Legatio e nell'In Flaccum coesistono. 
Il testo ha un contenuto singolare in riferimento alla produzione filoniana ${ }^{2}$ dal momento che si riferisce a circostanze autobiografiche e si pone immediatamente in correlazione all'In Flaccum in cui sono narrati avvenimenti in larga parte connessi.

D. T. Runia ha chiarito, riferendo anche esiti di approfondimenti di alcuni studiosi, le varie questioni interpretative inerenti a tale esordio: l'introduzione sembra infatti non connettersi specificamente con il resto del testo, come è evidente, in particolare, dalla cesura con i capitoli immediatamente successivi e inoltre introduce talune definizioni singolari non riscontrabili in altre opere dell'Alessandrino. ${ }^{3} \mathrm{Si}$ può ipotizzare che ci sia una lacuna? ${ }^{4} \mathrm{E}$, non avendo la possibilità di dimostrare la perdita di eventuali argomentazioni esistenti in precedenza, che valenza hanno i contenuti "preliminari" in cui prevalgono formulazioni teologiche? F. H. Colson aveva analizzato alcune concezioni espresse in apertura dell'opera rilevandone un nesso generico, non sorretto da una teoria coerente. L'accentuazione dell'esistenza di un tema focale nell'opera di Filone, l'introduzione cioè al grande Mistero del giudaismo, sostenuta da Goodenough, aveva indotto lo studioso a ribadire la presenza di tale contenuto anche nella premessa della Legatio, facendo di conseguenza propendere per l'ipotesi della perdita di uno svolgimento che avrebbe dovuto essere pertinente. E. Birnbaum si era concentrata, nella lettura della prefazione, sull'interpretazione semantica di Israele connesso unicamente in questo passo, con i Giudei. La capacità di "vedere Dio" non coincide con l'essere stati assegnati, "allotted" scriveva la studiosa, a Dio; i Giudei ricevono da Dio particolare attenzione, ed hanno un ruolo di intercessori nei confronti dell'umanità e con la loro osservanza della Legge superano la conoscenza raggiungibile con la filosofia. ${ }^{5}$ P. Borgen, ${ }^{6}$ in dissenso con alcune deduzioni di E. Birnbaum, affermava che questa introduzione ribadisce concetti di altri passi filoniani ed enfatizza la possibilità di rapportarsi misticamente a Dio, di salire verso l'alto, solo alle anime appartenenti al popolo di Israele. La conflittualità con antropologie di ambiti politeistici e l'opposizione all'ideologia e all'operatività di Caligola sono espresse chiaramente.

${ }^{2}$ P. Borgen (Philo of Alxandria, An Exegete for His Time. Leiden - New York - Köln 1997, 46-48) considera che gli scritti possano essere divisi in due gruppi: "Rewriting of the Laws of Moses" e "Exegetical Commentaries". La Legatio ad Caium e l'In Flaccum sono opere non corrispondenti a tali classificazioni.

${ }^{3}$ D. T. RUNIA (Philo of Alexandria, Legatio ad Gaium 1-7. In Neotestamentica et Philonica. Studies in Honor of P. Borgen. Ed. by D. E. AUNE - T. SEELAND - J. H. ULRICHSEN. Leiden 2003, 349-370) ha analizzato puntualmente il passo. In particolare, 350-351, ha segnalato le questioni inerenti all'etimo-

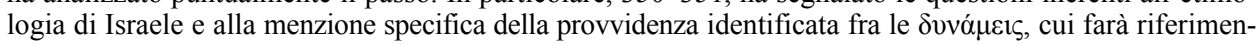
to in seguito. Significative sono le connessione che P. BORGEN (n. 2, 182-183), aveva indicato fra Legat. 3-7 ed alcuni passi presenti in De virtutibus, De praemiis e in Spec. 4. 132-238.

${ }^{4}$ RUNIA (n. 3) 352-353, pone il problema se i passi siano completi, come L. Massebieau aveva rilevato, o se sia ipotizzabile la presenza di una significativa lacuna, secondo la congettura di L. Cohn.

${ }^{5}$ E. BIRNBAum (The Place of Judaism in Philo's Thought. Israel, Jews, and Proselytes. Atlanta 1996, 189-192) analizza Legat. 1-7. Dopo avere posto la questione della ragione di questa introduzione alla Legatio, la studiosa scrive che Filone vuole mostrare ai lettori, sia Giudei che gentili che Dio guarda in particolare i Giudei anche attraverso le punizioni, come gli eventi narrati dimostrano. "As the suppliants' $\gamma \varepsilon$ vo $\varsigma$ which is dedicated to God, 'Israel' or the Jews exemplify something greater than philosophy, i.e., worship of God trough observance of His special laws. Laws, moreover, are made complete by providing for rewards and punishments, and these rewards and punishments come from God trough His powers».

${ }^{6}$ BORGEN (n. 2) 240-242. 
L'opportunità di un'analisi di questa parte introduttiva che non prescinda da valutazioni inerenti all'integrazione nel contesto di tutto il saggio è il punto di partenza dell'esame che D. T. Runia, dopo avere considerato le varie interpretazioni, elabora nel suo studio pubblicato in onore di P. Borgen.

Tale obiettivo è sicuramente condivisibile. Non escludendo la possibilità dell'esistenza di un contenuto più ampio e logicamente connesso, quale lettura può essere data dell'incipit? La valenza antropologico-teologica costituisce una premessa ideologica aprioristica rispetto alla narrazione specifica? La partecipazione esistenziale rende complessa la comprensione dei singoli avvenimenti e, di conseguenza ha reso necessaria una sospensione fra premesse teoriche ed esposizione di eventi? Qual è il focus di questi passi preliminari? L'esame di tali questioni comporta la possibilità di acquisire elementi per capire quali valenze Filone esprima nei confronti della realtà storica e del suo orizzonte interpretativo. Sarà opportuno considerare, fra l'altro, l'importanza dei contenuti che, non approfonditi in modo specifico in queste brevi annotazioni da Filone, rimandano a trattazioni presenti in altre opere, alle quali si potranno fare alcuni riferimenti esemplificativi.

L'input iniziale ha particolare rilevanza perché esprime tematiche fondamentali inerenti alla cognizione dell'antropologia a partire da un dato che descrive anche una condizione soggettiva. Filone, che quando scrive questo testo ha raggiunto la senilità, ${ }^{7}$ verifica in termini indiretti, il conseguimento di un autentico stato di anzianità. L'interrogativo ${ }^{8}$ pone un'alternativa:

Fino a quando noi vecchi continuiamo a restare bambini? L'età avanzata

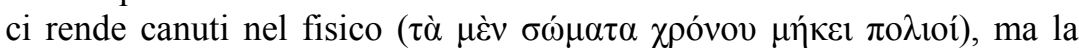
mancanza di discernimento lascia la nostra mente allo stadio dell'infan-

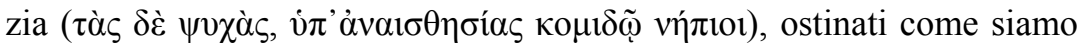
a considerare immutabile il caso, che è la cosa più vacillante del mondo, e soggetta a facili mutamenti la natura, che di tutte le cose è la più incrollabile (1). ${ }^{9}$

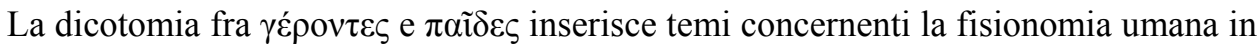
relazione allo scorrere del tempo esistenziale. Quale valenza assume questa distinzione? L'antecedenza nel pensiero di Filone ha spesso un valore di priorità essenziale che si segnala sia in ambiti metastorici o astratti sia nella stessa costituzione umana

${ }^{7} \mathrm{Si}$ suppone che la data probabile della nascita di Filone debba collocarsi intorno al 20 a.C. (CALABI, F.: Filone di Alessandria. Roma 2013, 15) o al 13 a.C. (come, riportando il punto di vista di J. Schwartz, scrive J. DANIÉLOU [Filone d'Alessandria. Roma 1991] nella traduzione del testo uscito nel 1958 in lingua originale). L'ambasceria a Roma si ritiene possa collocarsi nel 40 d.C. poiché il viaggio si sarebbe svolto nell'inverno fra il $39 \mathrm{e}$ il 40 . Arriva a queste conclusioni A. PELLETIER (Philon d'Alexandrie, Legatio ad Caium. Introduction, traduction et notes. Paris 1972, 29-30).

${ }^{8}$ Pelletier (n. 7) 60, n. 2, riportando il commento di A. G. Ross (Mnemosyne, 1935, 242) scrive che «cette prèmière phrase, étant le point de départ de out un raisonnement...ne doit pas s'entendre comme une interrogation oratoire, mais comme une affirmation adoucie».

${ }^{9}$ La traduzione dei passi della Legatio è tratta da KRAUS (n. 1). 
(Leg. II 6; Poster. 62; Poster. 91; Mutat. 94; Somn. II 135). ${ }^{10}$ In numerosi riferimenti è chiarito il significato attribuito alla polarità giovane/vecchio:

Il vecchio $\left(\pi \rho \varepsilon \sigma \beta v ́ \tau \varepsilon \rho \varsigma^{11}\right)^{11}$ nel vero significato del termine, non è ritenuto tale per la durata dei suoi giorni, ma per la sua vita degna di lode e perfetta. Coloro che hanno trascorso lungo tempo vivendo in rapporto al corpo, non raggiungendo una perfezione morale, sono dei bambini cresciuti, che non sono mai stati istruiti nelle conoscenze degne di una testa bianca. Ma colui che ama appassionatamente l'intelligenza ( $\varphi \rho o ́ v \eta \sigma ı \varsigma)$, la sapienza $(\sigma o \varphi i ́ \alpha)$ e la fede $(\pi i ́ \sigma \tau \iota \varsigma)$ in Dio, lo si potrà chiamare giustamente 'anziano' ( $\pi \rho \varepsilon \sigma \beta v ́ \tau \varepsilon \rho \circ \varsigma)$ nome che equivale a 'primo' (Abr. 271-272).

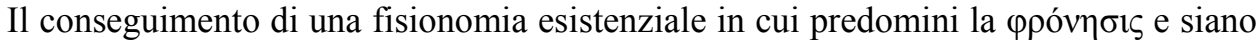

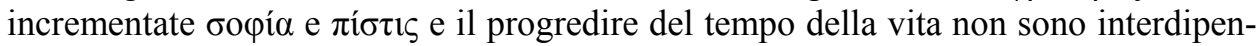
denti, hanno uno svolgimento differenziato e, di conseguenza, spesso, una distonia nella realtà globale dell'individuo stesso, come l'Alessandrino nota negativamente. Il $f o$ cus determinante è costituito dall'orientamento dell'anima che può comportare anche un sovvertimento nella possibilità del riconoscimento delle attribuzioni: "giovani" o "anziani": nei testi della Scrittura le due individuazioni, scrive Filone in de sobrietate 7, non sono collegate a valenze temporali: «In molti passi della Legge [Mosé] chiama 'giovani' uomini già avanti con gli anni, e d'altra parte chiama 'anziani' uomini che non sono ancora vecchi, dal momento che non guarda alla quantità degli anni o alla lunghezza o brevità del tempo, ma alle facoltà di un'anima che sia orientata in senso positivo o negativo». Un esempio è addotto anche nella Legatio e applicato a Tiberio: «Anzi, fin dalla giovinezza lo chiamavano il 'vecchio' ( $\pi \rho \varepsilon \sigma \beta v ́ \tau \eta \varsigma)$ per il rispetto che incuteva il suo acume» (142). Ma se può essere considerato positivo il raggiungimento delle caratterizzazioni proprie della vecchiaia in giovane età, è riprovevole il contrario.

La vita umana è quindi concepita secondo uno svolgimento in stadi la cui dinamica è considerata vantaggiosa. La prospettiva adeguata sembra infatti identificare un processo che giunge al compimento nell'acquisizione di della sapienza. La concezione sottesa alla storia individuale è quindi, secondo Filone, positiva. L'accento è posto

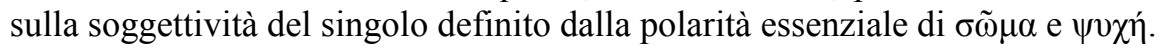

${ }^{10}$ Mazzanti, A. M.: Filone di Alessandria. In Senectus. La vecchiaia nell'antichità ebraica e cristiana. U. MATTIOLI (a cura di) con la collaborazione di A. CACCIARI e V. NERI. Bologna 2007, 99-109, partic. 100 .

Si legga in Leg. II 6: «Come da parte dei medici e degli studiosi della natura più preparati si sostiene che il cuore si sia formato prima del corpo nel suo complesso, quasi fosse il fondamento o la carena di una nave, su cui si costruisce il resto del corpo [...] così si ritiene che la parte dominante dell'anima sia più vecchia $(\pi \rho \varepsilon \sigma \beta\langle ́ \tau \varepsilon \varepsilon \rho \nu)$ dell'anima nel suo complesso, e la parte irrazionale sia più giovane [...]. Ora la parte irrazionale è la sensazione e le passioni che ne vengono generate [...]. Così questo aiuto è più giovane, $\mathrm{e}$, come è naturale, è generato».

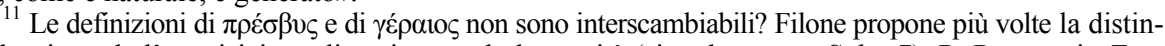
zione che riguarda l'acquisizione di sapienza o la longevità (si veda per es. Sobr. 7). R. RADICE in Tutti $i$ trattati 654, n. 13 afferma che il termine $\pi \rho \varepsilon ́ \sigma \beta \nu \varsigma$ assume un senso eminentemente morale, mentre

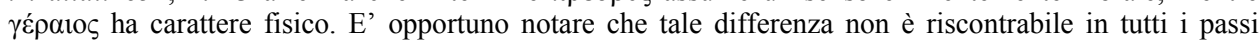

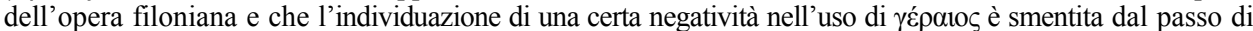

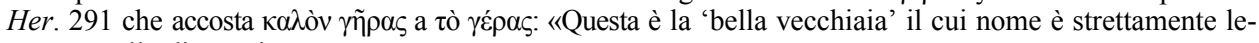
gato a quello di premio».

Acta Ant. Hung. 56, 2016 
La riflessione sulla struttura antropologica si approfondisce nel passo successivo in cui, a fronte dell'incapacità di cogliere l'ordine della natura nelle vicende umane,

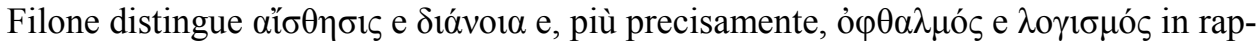
porto alla finalità gnoseologica.

La ragione è questa: giudichiamo ${ }^{12}$ il presente senza alcuna preveggenza del futuro, affidandoci alla fallacità dei sensi anziché seguire il sicuro criterio dell'intelletto. Con gli occhi si colgono solo gli oggetti visibili e presenti, mentre la ragione penetra nel campo delle cose invisibili e future, la cui visione richiede un occhio più acuto di quello fisico. Questa visione è offuscata in alcuni di noi dagli eccessi del vino o della gola, in altri dall'ignoranza, che è il peggiore dei mali (2).

L'inganno della capacità sensitiva legata al presente è considerata in antitesi

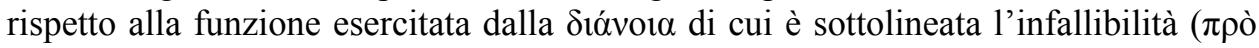
$\delta 1 \alpha v o i ́ a \varsigma \alpha \grave{\alpha} \pi \lambda \alpha v o v \tilde{\varsigma})$. La prospettiva cambia nella successiva differenziazione di ò $\varphi$ -

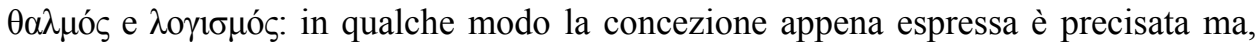
nel contempo, sono delineati altri elementi. Mentre gli occhi percepiscono solo il presente, le realtà cioè visibili e tangibili, la razionalità, puntualizza Filone, coglie le cose invisibili ed è in grado di intuire il possibile sviluppo futuro; eppure, questa mag-

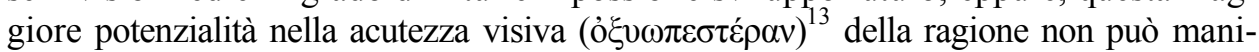
festarsi perché i vizi legati alla gola e soprattutto l'd̆ $\mu \alpha \theta i ́ \alpha$ ne oscurano l'esercizio. Esempio di tale negatività è, nelle pagine successive, lo stesso Gaio che

si era lasciato andare alla dissipatezza. Eccesso nel bere, cibi arzigogolati, un appetito insaziabile anche quando lo stomaco era pieno da scoppiare, bagni caldi fuori tempo, vomiti e subito dopo ubriacature e ingestione di nuove ghiottonerie, lascivie con ragazzi e donne, e ogni altro vizio che distrugge l'anima e il corpo e i legami che li tengono uniti: fu tutto un assalto simultaneo. La moderazione trova il suo compenso nella salute e nella forza, l'incontinenza nella debolezza e nella malattia che può portare alla morte (14). ${ }^{14}$

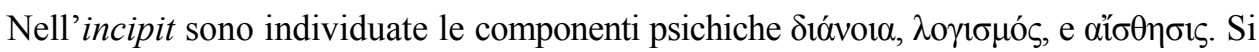
coglie un accenno alla concezione tripartita dell'anima? E' opportuno osservare che

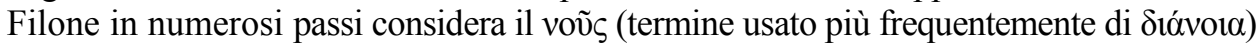

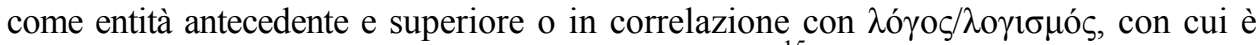
anche interscambiabile e, come scrive J. Whittaker, ${ }^{15}$ «expands frequently the cou-

\footnotetext{
${ }^{12}$ Il verbo usato è $\beta \rho \alpha \beta \varepsilon v ́ \omega:$ è applicata la metafora delle gare sportive. PELLETIER (n.7) ha tradotto: «La cause en est dans le fait que nous donnons le prix au present, dans notre imprrévoyance de l'avenir».

${ }^{13} \mathrm{Si}$ tengano presenti, per converso, le metafore della luce e del sole usate per delineare il $\Lambda$ ó $\gamma o \zeta$. F. CALABI (Metafore del logos in Filone di Alessandria. In RADICE, R. - VALVO, A. [a cura di]: Dal Logos dei Greci e dei Romani al Logos di Dio. Ricordando Marta Sordi. Milano 2011), ne ha considerato le valenze, sottolineando la possibilità della visione degli occhi dell'anima, seppure in modo parziale. L'uso del comparativo nella citazione è peraltro indicativa.

${ }^{14}$ L'accentuazione sulla vita dissipata e sul dominio dei sensi nella vita di Gaio è riaffermato in Legat. 168.

${ }^{15}$ J. WhitTAKeR (How to Define the Rational Soul? In LÉVY, C. [éd.]: Philon d'Alexandrie et le langage de la philosophie. Turnhout 1998, 229-253), ha analizzato i termini usati dall'Alessandrino per definire le componenti dell'anima cogliendo le relazioni con concezioni prevalentemente platoniche e stoiche.
} 


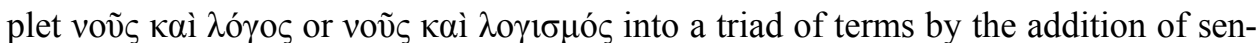
se-perception ( $\alpha \grave{\imath} \sigma \theta \eta \sigma ı)$ )». Nell'esegesi del racconto genesiaco l'essenza del voũ $\varsigma$ è con-

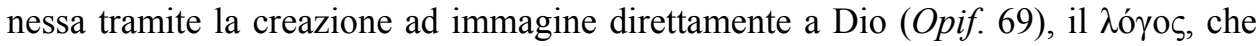
compare anche in relazione a tematiche etiche (Opif. 73), è identificato con la parte egemonica dell'anima nell'interpretazione della formazione dell'uomo plasmato in Gen.

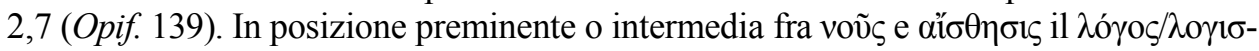
$\mu$ ó $\varsigma$ svolge comunque una funzione valutativa. Eppure, in tale dinamica, si intromette

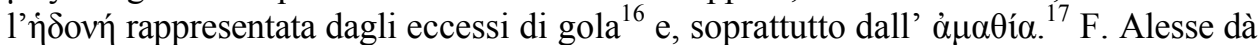
rilievo al paragone espresso da Filone, che è influenzato dall'Alcibiade I, fra il ruolo dell'intelletto nell'attività della conoscenza e la percezione visiva, ripreso in vari passi. ${ }^{18}$

Il tema enunciato trova riscontro successivamente nel testo, in un passo in cui l'alternativa voṽ $/ \alpha i ̂ \sigma \theta \eta \sigma ı s$ è messa in rapporto in modo problematico con la concezione, riproposta più volte in ambito genesiaco, sulla differenza dei sessi. ${ }^{19}$

La modalità dell'uso del ragionamento in relazione all'età e all'educazione è chiarito nelle considerazioni personali espresse dall'Alessandrino dopo il primo incontro con Caligola. Alle congratulazioni per la supposta benevolenza concessa, Filone scrive:

$\mathrm{Ma}$ io che credo di vederci più chiaro per la mia età $\mathrm{e}$ in genere per la mia educazione $(\pi \alpha 1 \delta \varepsilon i ́ \alpha)$, ero più cauto nell'interpretare un atteggiamento di cui gli altri si rallegravano (182),

ed aggiunge che le sue deduzioni nascevano dal concentrarsi sul ragionamento (’่v $\alpha$ -

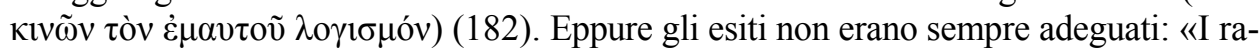

\footnotetext{
${ }^{16}$ Indicativo, fra altri, il passo di Leg. III 156 in cui Filone, riferendosi alla sua esperienza personale, riferisce quale sia la modalità adeguata della sua partecipazione ai banchetti.

${ }^{17}$ Su questi temi si rimanda a MAZZANTI, A. M.: Il $\lambda o ́$ os nell'antropologia di Filone d'Alessandria. Considerazioni sulla creazione dell'uomo in De opificio mundi e in Legum Allegoriae. In RADICEVALVO (n. 13) 85-101.

${ }^{18}$ ALESSE, F.: Il luogo del nous: Alcuni aspetti dell'antropologia di Filone Alessandrino. In MAZZANTI, A. M. - CALABI, F. (a cura di): La rivelazione in Filone di Alessandria: natura, legge, storia. Atti del VII Convegno di Studi del Gruppo Italiano di Ricerca su Origene e la Tradizione Alessandrina. Villa Verucchio 2004, 105-122, 106. Si noti la designazione del voṽ come pupilla nell'occhio in De opificio mundi 66.

${ }^{19}$ Significativa, in relazione alla tematica voũ $\varsigma, \lambda o ́ \gamma o \varsigma, \alpha i ̋ \sigma \theta \eta \sigma \iota \varsigma$ il passo, nel discorso che Agrippa pronuncia rivolgendosi a Caligola e facendo riferimento a Giulia Augusta (319-320): «Seguendo l'esempio di un simile maestro di pietà anche la tua bisavola, Giulia Augusta, adornò il tempio di ampolle d'oro, di coppe da libagione e di molti altri preziosi doni votivi. Quale dovette essere anche il suo pensiero di fronte al fatto che nel Tempio non ci fosse alcuna immagine? In genere la capacità critica delle donne è più debole quando non si tratti di cose sensibili, perché non sono in grado di afferrare concetti astratti. Ma lei si distinse anche in questo, come in tutto il resto, dal suo sesso. L'educazione perfetta aveva accentuato le sue inclinazioni naturali, e l'aveva resa superiore nella pratica, sviluppando in lei un modo di ragionare mas-

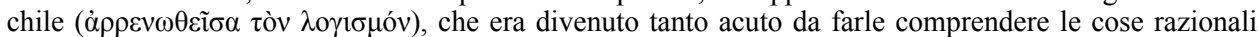

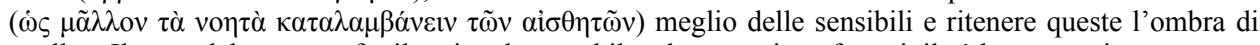
quelle». Il tema del rapporto fra il razionale maschile e la sensazione femminile è ben noto sia con accenti che ne considerino la differente valutazione secondo una gerarchia di priorità temporale e ontologica (Quaest. Gen. I 25; I 37; I 45-47; Leg. II 38 ss.; Fug. 188; Cher. 41), sia con formulazioni che indichino

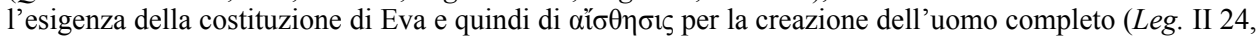
40-41; III 222). Si veda su questo argomento MAZZANTI, A. M.: Motivazioni protologiche nell'antropologia di Filone di Alessandria, con riferimento al tema della distinzione dei sessi. In BIANCHI, U. (a cura di): La tradizione dell'enkrateia. Motivazioni ontologiche e protologiche. Roma 1985. Interessante coglie-

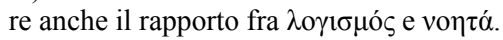

Acta Ant. Hung. 56, 2016 
gionamenti che andavo facendo con me stesso mi mettevano in grande agitazione e non riuscivo a trovare pace né giorno né notte» (184). Filone infatti riconosce (per inciso, in un passo in cui narra la decisione attuata da Petronio) che le situazioni gravi e improvvise impediscono talora di usare la ragione in modo adeguato (221).

L'incapacità della mente umana di giungere alla conoscenza è ripresa ulteriormente in una valutazione più generica riguardante l'errore commesso dai popoli nel riporre la speranza nella positività dell'impero sotto la guida di Caligola:

La mente umana (ó $\alpha \dot{v} \theta \rho \omega ́ \pi t v o \varsigma$ voũ $\varsigma$ ) è cieca nel valutare il suo vero utile, perché è capace solo di congetture e di supposizioni e non sa prendere a guida la conoscenza $\left(\dot{\varepsilon} \pi\llcorner\sigma \tau \eta \dot{\mu \eta})^{20}(21)\right.$.

Il sentimento stesso e non il ragionamento influenza il criterio usato dai popoli nei confronti delle proprie istituzioni, considerate positive, pur non essendo in verità tali (277).

$\mathrm{E}$, a fronte delle difficoltà della valutazione autentica del $\lambda$ ó $\gamma$ os, interviene la Legge. Filone, ipotizzando quali possano essere le cause delle obiezioni, attesta la possibilità, successivamente allo smarrimento e all'ansia provocati da notizie drammatiche, di comprendere il significato degli avvenimenti e di basare la speranza della salvezza (e quindi la visione del futuro) sullo studio della Legge e quindi sulla memoria. La conoscenza della Legge, in aggiunta alla natura, è una guida per la maggior parte degli uomini, e la sua comprensione, scrive J. W. Martens in un'analisi approfondita sul tema, conduce alla cognizione del trascendente e insieme alla sequela esistenziale. ${ }^{21}$

"I casi sono due: o tu non hai sentimenti genuini da uomo nobile (àvopò $\varsigma$

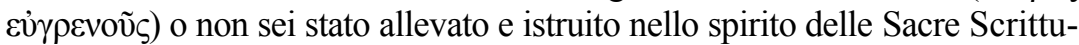

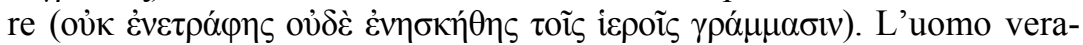

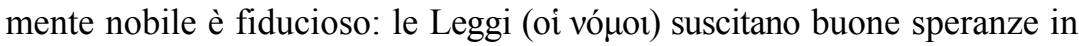
chi non si limita a studiarle in superficie". Forse queste sventure sono un mezzo per mettere alla prova la nostra generazione, per scoprire a quale grado di virtù si trovi, se sia stata educata a sopportare le avversità con ragio-

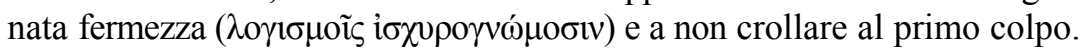
Ogni soccorso umano ci abbandona, e lasciamo che ci abbandoni. Ma nelle nostre anime rimanga indistruttibile la speranza in Dio, nostro Sal-

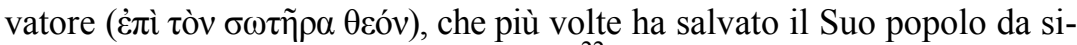
tuazioni estreme e disperate $(195-196) .{ }^{22}$

Cosa significa essere un uomo nobile? L'accezione di un dato genetico evoca immediatamente il giudizio che Filone esprime nei confronti di Ottaviano Augusto «Che dire di colui che in tutte le virtù trascese la natura umana (

\footnotetext{
${ }^{20}$ PeLletier (n. 7) 79, n. 4 traduce con "connaissance objective" ricordando che il vocabolo identifica la "science de l'être".

${ }^{21}$ In One God, one Law. Philo of Alexandria on the Mosaic and Greco-Roman Law. Boston-Leiden 2003, 126-130, J. W. MARTENS esamina la rilevanza fondamentale della Legge di Mosè e ne afferma l'importanza in relazione alla fede. Ios. 195.

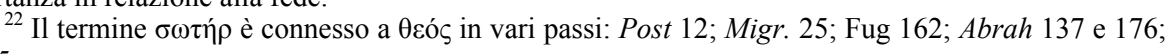


(143), così come il rilievo attribuito a Petronio che, avendo riunito «tutte le sue facoltà

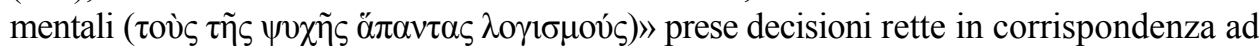

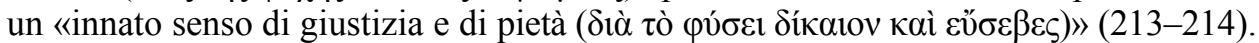
La formazione nel tempo alle Scritture e l'approfondimento della Legge sono ulteriori elementi determinanti perché emerga stabilmente la speranza: $:^{23}$ «Gli Ebrei, scrive Filone, considerano le loro Leggi responsi oracolari dati da Dio ed essendo educati in questa convinzione fin dalla più tenera infanzia, portano impresse nel cuore le immagini dei Comandamenti» (210). La negatività dell'ỏ $\mu \alpha \theta^{\prime} \alpha$ come causa di male è già stata affermata.

Filone si sofferma successivamente sul contenuto fondamentale. Espresso nel prologo, l'esito del processo cognitivo, la concezione della realtà, o meglio dell'origine

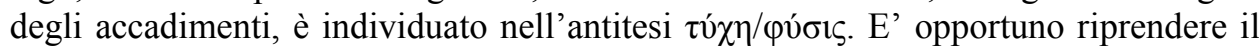
testo dell'incipit che (attribuendo la scelta giudicata negativa ai vecchi rimasti bambini) recita

ostinati come siamo a considerare immutabile il caso, che è la cosa più vacillante del mondo, e soggetta a facili mutamenti la natura, che di tutte le cose è la più incrollabile. Sembra si faccia al giuoco del tavoliere con questo nostro spostare e scambiare i termini della realtà, nella ferma convinzione che le vicende casuali siano più stabili dei caratteri inerenti alla natura e che l'ordine naturale sia più labile degli scherzi del caso (1-2).

La nozione di $\tau u ́ \chi \eta$, collegata all'immagine del gioco, derivata in origine da Eraclito, ${ }^{24}$ è ribadita in altri passi ${ }^{25}$ da Filone. L'instabilità degli eventi inerenti sia a persone sia al popolo giudaico sia alla situazione dell'impero è ampiamente documentata nella narrazione e la valutazione sul predominio della casualità nella realtà può apparire consequenziale. D. T. Runia cita i passi in cui nella Legatio sono espressi termini inerenti a $\tau$ $u ́ \chi \eta .{ }^{26}$ Secondo L. Troiani, le affermazioni del proemio chiariscono che «la storia di cui sono protagonisti i politici è qui, in coerenza con il suo pensiero e la sua filosofia, relegata nell'ambito delle cose instabili ed evanescenti». Valutazioni analoghe sono espresse anche in De Iosepho. La storia politica, scrive concludendo lo studioso, è utilizzata dall'Alessandrino «come strumento per delineare meglio i percorsi della sua speculazione filosofica». ${ }^{27} \mathrm{E}$ ' necessario riconoscere la profonda di-

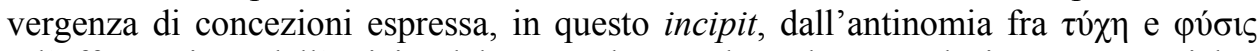
e l'affermazione dell'unicità del senso che non ha valenze esclusivamente teoriche, ma è l'esito del giudizio su accadimenti che hanno risvolti esistenziali personali e che

\footnotetext{
${ }^{23}$ Filone ritiene che esistano due razze di uomini, il perfetto e il progrediente? DANIÉLOU (n. 7) 222, affermava che, in difformità rispetto al dualismo antropologico gnostico, l'Alessandrino considera il cammino e l'acquisizione della saggezza che è di origine divina due tappe differenti dell'esistenza individuale e ne notava la relazione con formulazioni stoiche. La citazione di Congr. 36-38 e di Deus 155-160 confortano tale lettura.

${ }_{25}^{24}$ Fr. B52. Si veda Platone, Leggi 903b.

${ }^{25}$ RuNIA (n. 3) 357, cita i passi di Mos 1, 31-32 e Ios. 125-147 in cui è ripresa l'immagine del gioco di dadi.

${ }^{26}$ RUNIA (n. 3) 358-359.

${ }^{27}$ TROIANI, L.: Natura e storia politica in Filone d'Alessandria. In MAZZANTI-CALABI (n. 18) 1-8.
} 
l'autore ritiene siano rivelativi: «Ma la circostanza attuale e le molte e importanti questioni definitesi nel corso di essa varranno a convincere anche gli increduli» (3).

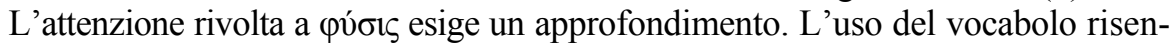
te di influssi specifici significativi. Il termine, che, secondo l'analisi semantica di M. Morani, ${ }^{28}$ aveva in origine il valore di "potenzialità di accrescimento" assunse anche il senso di "situazione determinata, carattere". Il coesistere dei due valori comportò di conseguenza un processo di generalizzazione, la definizione cioè di una realtà universale, e favorì il passaggio, attestato in Aristotele, dalla definizione di natura data all'insieme del creato a quella inerente alla forza stessa che ha generato la realtà. Ne derivò pertanto l'identificazione con Dio. ${ }^{29}$ In Filone, come scrive C. Anderson in una interessante monografia sulle concezioni inerenti al mondo fisico nell'opera filoniana, ${ }^{30}$ questi elementi sono presenti, in particolare le accezioni stoiche che, influenzate dall'aristotelismo, avevano posto l'accento sulla razionalità dell'atto creativo e, insieme, sull'aspetto dinamico, pervasivo. Permane discriminante la nozione della separa-

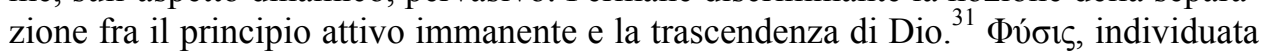
nella realtà delle stagioni in Legat. 190 e in talune componenti della persona umana in Legat. 56 e $229,{ }^{32}$ è riconosciuta come carattere essenziale che distingue il mortale dall'immortale in Legat. $91^{33} \mathrm{e}$ in Legat. $320^{34} \mathrm{e}$ come natura di Dio in Legat. $310{ }^{35}$ Lo studioso cita questi passi enumerandoli fra quelli che esemplificano il potere crea-

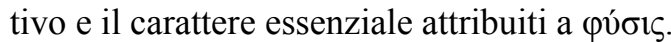

E' importante notare che nell'accentuare la differenziazione fra la realtà divina e quella umana più volte Filone mette in risalto, in vari passi dell'opera, la pretesa di Gaio di ritenersi divino. L'Alessandrino gli attribuisce l'intenzione di poter valicare i li-

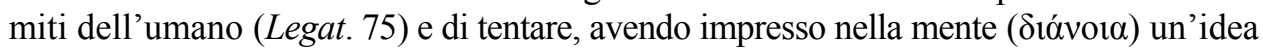
non conforme a verità, l'assimilazione prima ai cosiddetti semidei, poi a divinità maggiori ( $«$ Gaio cadde in preda a una frenesia selvaggia e delirante fino al punto che gli sembrarono troppo poco persino i semidei e si propose mete più alte» 93) tramite l'assunzione di aspetti esteriori e di atteggiamenti da figurante teatrale mentre, nel contempo, risulta evidente l'antitesi nel comportamento per quanto riguarda le virtù e le prerogative specifiche ${ }^{36}$ degli stessi dei $^{37}$ (Legat. 76-114).

\footnotetext{
${ }^{28}$ MORANI, M.: Physis e natura, divagazioni semantiche su una parola. In MAZZANTI, A. M. (a cura di): Il logos di Dio e il logos dell'uomo. Concezioni antropologiche nel mondo antico e riflessi contemporanei. Milano 2014, 7-27.

${ }^{29}$ MORANI (n. 28) 15-16.

${ }^{30}$ ANDERSON, C. A.: Philo of Alexandria's Views of the Physical World. Tübingen 2011.

${ }^{31}$ ANDERSON (n. 30) 108. Si veda anche J. W. MARTENS (n. 21) 86, ripreso anche da Anderson, che nota come Filone assuma concezioni stoiche senza contraddire il giudaismo.

${ }_{33}^{32}$ ANDERSON (n. 30) 109, 110, n. 49 e 110.

${ }^{33}$ ANDERSON (n. 30) 114, n. 77.

${ }_{35}^{34}$ ANDERSON (n. 30) 116, n. 88.

${ }^{35}$ ANDERSON (n. 30) 114, n. 74.

${ }^{36}$ Da notare, fra l'altro le affermazioni inerenti ad Ares: «E' cosa risaputa che il potere di Ares -

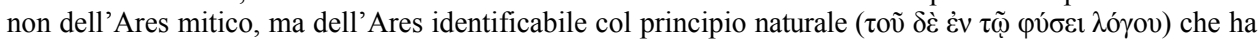
per attributo specifico il coraggio virile - è un potere che mira ad allontanare il male» (112).

${ }^{37}$ Rilevante la lunga descrizione dei comportamenti di Caligola. L. LUGARESI (Il teatro di Dio. Il problema degli spettacoli nel cristianesimo antico (II-IV secolo). Brescia 2008, 485) scrive, commentando
} 


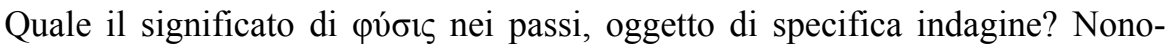
stante la complessità dell'identificazione nelle formulazioni che sono espresse nei vari scritti e che Anderson analizza in profondità, ${ }^{38}$ la natura, oltre che come potere creativo, è intesa come natura universale in connessione con Dio, di cui manifesta l'esisten$\mathrm{za},{ }^{39}$ è principio di ordine cosmico e di normatività, in relazione con la legge mosai-

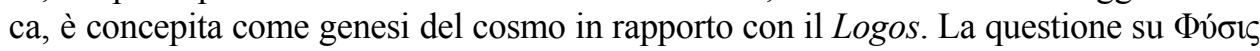
come metonimia di Dio, elemento focale per l'interpretazione dell'orizzonte concettuale cui fa riferimento Filone, è oggetto di pareri controversi da parte degli studiosi. Il problema è rappresentato dalla possibile negazione della trascendenza, in conformità con concezioni stoiche che emergono chiaramente in taluni passi. Filone, secondo Anderson, non subisce influssi così profondi e si distanzia sia nel negare l'identifica-

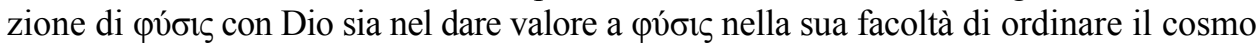
e la vita umana in corrispondenza alla Legge mosaica.

Nell'accentuazione della contrapposizione fra $\tau \dot{\chi} \chi \eta$ e $\varphi v ́ \sigma ı \varsigma$, caratterizzata da stabilità, sicurezza, la natura è individuata come potenza creativa e ordine del cosmo nella sua corrispondenza essenziale a Dio di cui rivela la presenza operativa o, meglio, l'azione benefattrice.

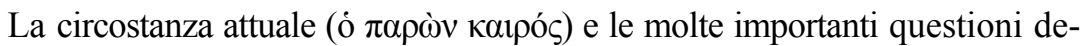
finitesi nel corso di essa varranno a convincere anche gli increduli, che non ammettono esista una provvidenza divina nei confronti dell'umanità $\mathrm{e}$ in modo speciale del popolo supplice, scelto per elezione dal Padre e Re e Creatore del mondo (3).

Gli eventi sono significativi e di per sé persuasivi nella loro successione storica. La visione non si fonda su astrazioni ma su riscontri di fatti.

La certezza acquisita riguarda la provvidenza: rivolta a tutti, è particolarmente

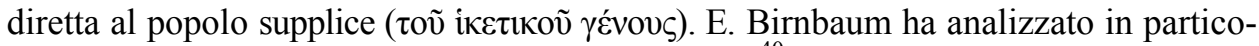
lare le definizioni con cui Filone nomina i Giudei. ${ }^{40} \mathrm{Il}$ termine $\gamma \varepsilon \dot{\varepsilon} v$ s, ripreso in $\mathrm{Le}$ gatio 178, 201, 346 sembra definire la razza ebraica in toto a differenza di है $\theta v o \varsigma$ che qualificherebbe i Giudei di Alessandria. L'azione della supplica, pur non essendo espressa in altri passi tramite l'aggettivo usato in questo prologo, è più volte enunciata nella forma sostantivata: supplicanti sono chiamati i Leviti e i proseliti, come supplicanti di Dio e della Legge. Tale designazione enfatizza, secondo la studiosa, l'esistenza di una relazione fra uomo e Dio e non prioritariamente l'appartenenza ad una nazione. ${ }^{41}$ D. T. Runia, che mette in evidenza gli elementi di comparazione riscontrabili

questi passi: «Sempre legata alla rappresentazione della regalità è la critica spietata che Filone muove alla pretesa di divinizzazione avanzata da Caligola. Essa viene infatti classificata sotto la categoria, assolutamente degradante, del teatro: nel suo impudente tentativo di accreditare presso i sudditi la propria identità divina, l'imperatore si comporta in effetti come un attore che recita la parete degli déi sul palcoscenico». Lo studioso aggiunge che emerge nell'esposizione quel tratto della "molteplicità" come caratteristica negativa del corpo dell'attore.

${ }^{38} \mathrm{Si}$ vedano i capitoli 5 e 6 in ANDERSON (n. 30).

${ }^{39}$ ANDERSON (n. 30) 121-122 e 128

${ }^{40}$ BiRnBAUM (n. 5) 61-127.

${ }^{41}$ Birnbaum (n. 5) 106, n. 22. 
nella relazione con Dio e con Gaio, ${ }^{42}$ fa presente la condizione di richiesta dei protagonisti della Legatio che, nella narrazione, respinti dall'imperatore, si rivolgono al vero Dio (366). E' necessario notare che "l'essere supplice" indica uno status posto in corrispondenza all'elezione di Dio di cui sono precisati la paternità, la regalità e l'esse-

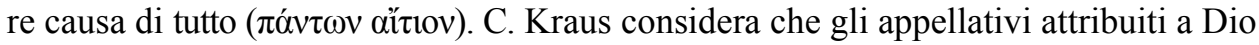
sono legati all'idea di creazionismo cui corrisponde il concetto di provvidenza, come si legge anche in De opificio 9-10 e in De praemiis $41 .^{43}$ All'individuazione corrisponde la dinamica esistenziale. Oggetto di approfondimenti specifici è l'identificazione di "Israele" nel passo successivo:

Questo popolo è chiamato con vocabolo caldaico Israele; tradotto in greco il termine significa "colui che vede Dio". (4).

Significative le conclusioni a cui è giunta E. Birnbaum che ha esaminato a lungo le deno-

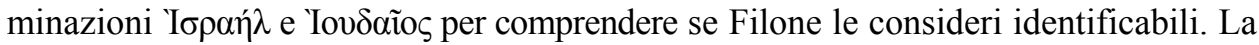
connessione fra Israele e il popolo dei Giudei nello stesso passo e la spiegazione etimologica, presenti solo in questo brano della Legatio, che la studiosa ritiene rivolto a Giudei e a non Giudei, possono essere interpretato come «his [cioè di Filone] wish to impress his readers by depicting the Jews as those who embody the ideal of seeing God, which "Israel" represents»». ${ }^{44} \mathrm{P}$. Borgen, nel considerare la caratterizzazione della nazione giudaica precisa i nessi esistenti fra Legatio 3-7 e altre opere filoniane. In particolare lo studioso collega la designazione di Israele con l'attribuzione del nome di "colui che vede Dio" a Giacobbe nell'esegesi di De Praemiis 36ss.; ne consegue l'individuazione del popolo giudaico. ${ }^{45}$ D. T. Runia, precisando i termini del dibattito sulle concezioni inerenti al "vedere Dio" in questo brano, esamina il significato in relazione alle formulazioni successive. ${ }^{46}$

Il tema del vedere, già esaminato nella distinzione fra la potenzialità di funzio-

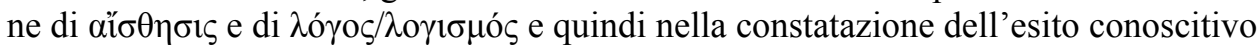
nei confronti degli avvenimenti della realtà, è svolto nella constatazione dell'obiettivo più profondo, più prezioso sia per l'individuo che per il popolo. L'esigenza educativa, riproposta nel paragone della relazione con anziani, maestri, capi e genitori, è di nuovo affermata come fondamentale ${ }^{47}$ per protendere lo sguardo verso la visione del non creato, del divino.

Ora la visione di Dio mi sembra il più prezioso tra tutti i privilegi privati o pubblici. Se la vista degli anziani, dei maestri, dei capi, dei genitori

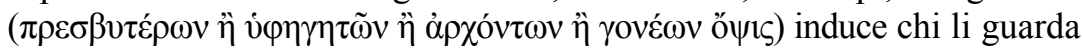
al ritegno, alla compostezza, al desiderio di una vita retta, quale saldezza di

\footnotetext{
${ }^{42}$ RUNIA (n. 3) 361.

${ }^{43}$ KRAUS, C.: La presenza di Dio nella storia secondo Filone di Alessandria. In MAZZANTI-CALABI (n. 18) 9-13.

${ }^{44}$ BirnBAum (n. 5) 105-108, 189-192 e 224.

${ }^{45}$ Borgen (n. 2) 182-183.

${ }^{46}$ RUNIA (n. 3) 361-363.

${ }^{47}$ L'importanza dell'educazione per Filone è precisata più volte: è rilevante nell'indurre alla virtù $\mathrm{o}$ alla $\dot{\alpha} \sigma \varepsilon ́ \beta \varepsilon 1 \alpha$ : Sacrif. 15; Virt. 178; Her. 295.
} 
virtù e di perfezione morale non scopriremo noi in anime, che sono state educate a spingere lo sguardo al di là del creato e a contemplare il non creato e divino, il sommo bene, la suprema bellezza, felicità e beatitudine, l'Essere che, a voler tener fede alla verità, è migliore del bene, più bello del bello, più beato della beatitudine, più felice della felicità stessa, più perfetto di ogni possibile perfezione, ammesso che ne esista una più compiuta di queste?

Il divino è identificato secondo i termini assoluti della via eminentiae: è affermata la supremazia dell'Essere rispetto alle stesse perfezioni concepite per astrazione. ${ }^{48}$ Ed è significativo che sia il $\lambda o ́ \gamma o \zeta$, e non il voũ $\varsigma$ come si potrebbe ipotizzare in relazione alle distinzioni antropologiche più volte formulate da Filone, in particolare nell'esegesi della creazione, ${ }^{49}$ che, nel tentativo di rapportarsi a Dio, sperimenta la sua impotenza e rende evidenti le caratterizzazioni che ne rivelano la distanza ontologica: conseguente è allora la via negationis che radicalizza tale impossibilità conoscitiva ed espressiva. ${ }^{50}$ Le valenze del termine $\lambda$ ó $\gamma$ $\zeta$ che dalla sfera semantica del dire passa a sviluppare significati correlati all'attività razionale ${ }^{51}$ sono attestate contemporaneamente in questo passo, come sottolinea D.T. Runia, soffermandosi sulle traduzioni del testo. ${ }^{52}$

Perché il $\lambda$ ó $0 \varsigma$ non può arrivare a Dio, che è assolutamente intangibile e inaccessibile, ma arretra e precipita, incapace di trovare termini atti a definire per gradi non dico l'Essere Supremo - neppure il cielo intero se si trasformasse in voce articolata troverebbe le espressioni adeguate - ma i poteri che di quell'Essere sono le guardie del corpo ( $\tau \tilde{\omega} v \delta o \rho v \varphi o ́ \rho \omega v$

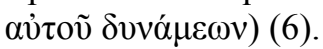

Non solo l'essenza di Dio è inaccessibile (significativa è l'iperbole espressa in questo passo), ${ }^{53}$ ma anche quella delle $\delta v v \alpha ́ \mu \varepsilon ı \varsigma$ si colloca su un piano trascendente. ${ }^{54}$

${ }^{48}$ «Perché se qualcosa si aggiungesse a Dio, questo dovrebbe essere o maggiore, o minore, o uguale A Lui. Ma nulla c'è che sia uguale o maggiore di Dio e, d'altra parte, l'aggiungersi di qualcosa che gli fosse inferiore non Lo accrescerebbe di nulla, anzi, semmai, Lo sminuirebbe» (Leg. II 39) (trad. R. Radice in Filone, Tutti i trattati del Commentario allegorico alla Bibbia. A cura di R. RADICE, con la collaborazione di G. Reale, D. Kraus RegGiani e C. MAzzarelli. Introduzione di G. Reale. Milano 1994).

${ }^{49} \mathrm{Si}$ veda Opif. 69-71 in cui Filone commenta Gn.1,26-27: la creazione dell'uomo "ad immagine" individua la genesi del voũc.

${ }^{50} \mathrm{Nel}$ momento in cui si dà un'interpretazione indiretta sulla natura divina, come scrive F. CALABI, (Conoscibilità e inconoscibilità di Dio in Filone di Alessandria. In CALABI, F. [a cura di]: Arrhetos Theos. L'ineffabilità del primo principio nel medioplatonismo. Pisa 2002, 35-54, 47) citando Vanderlin, la via eminentiae si rapporta alla via negationis e, nel contempo, si annulla.

${ }^{51} \mathrm{Si}$ veda il saggio di MORANI, M.: La semantica di logos fra greco pagano e greco cristiano. In VALVO-RADICE (n. 13) 29-64. Lo studioso esamina i significati assunti dal termine logos a partire dalla radice indoeuropea nella letteratura greca classica, nell'incontro con la cultura semitica e nel Nuovo Testamento.

${ }_{52}^{52}$ RUNIA (n. 3) 365

${ }^{53}$ CALABI: Conoscibilità (n. 50) 38-40, pone la questione della non nominabilità di Dio o dell'impossibilità per l'uomo di conoscerne il nome. Dio non ha nome o non lo rivela? L'impossibilità di conoscere l'essenza di Dio, determina l'impossibilità di conoscere il nome. All'impossibilità di vedere Dio (Mutat. 9) corrisponde l'impossibilità di conoscere il nome di "Colui che è" (Mutat. 11).

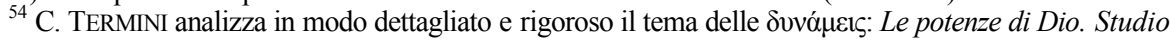
su dúvauı in Filone di Alessandria. Roma 2000. A p. 46 commentando Spec. 1.32-50 in cui compaiono alcu- 


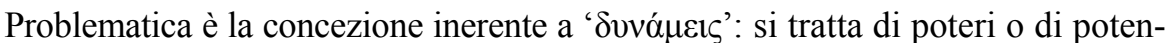
ze? Concepibili in vari passi come epifanie del divino, individuabili dall'uomo nel cammino ascetico, secondo una polinomia che risente fortemente di influssi ellenistici, sono più difficilmente identificabili, negli interventi attribuiti in ambito antropogonico. Sembrano essere collocabili in una struttura sia orizzontale che verticale

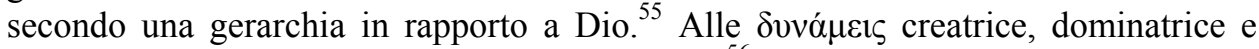
provvidenziale, menzionata solo in questo passo, ${ }^{56}$ se ne affiancano altre non distinte nella denominazione.$^{57}$ La metafora della guardia del corpo, applicato alle potenze sembra configurare delle entità a sé stanti. C. Termini, citando questo passo insieme ad altri in cui ricompare la stessa attribuzione, ritiene che tale definizione non sia

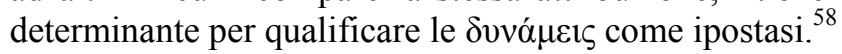

Intendo dire il potere creativo, il potere regale, il potere provvidenziale e gli

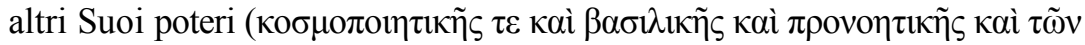
$\alpha \ddot{\alpha} \lambda \lambda \omega v)$ benefici e punitivi, premesso che anche i poteri punitivi devono essere inclusi tra quelli benefici, non solo in quanto parti delle leggi e delle norme (la legge per sua natura è perfetta se include ambedue gli elementi: la premiazione dei buoni e la punizione dei malvagi), ma anche perché la punizione serve di monito e spesso mette sul giusto cammino gli autori stessi di una colpa o, se non i colpevoli, sicuramente chi si trova a contatto con loro. Le punizioni inflitte agli altri rendono infatti migliore la moltitudine, perché la inducono a temere una pena analoga (7).

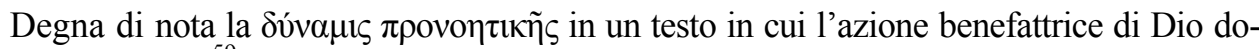
mina. P. Frick ${ }^{59}$ analizza le caratterizzazioni della provvidenza nella Legatio: descritta come aiuto divino che si ritiene interverrà secondo modalità già sperimentate in situazioni estreme e disperate (196) introduce alla speranza. Il suo operare inaspettato (367) sollecita alla gratitudine e si giustifica in relazione alla riparazione di un torto (220) e come giustizia (336).

La pluralità delle teofanie si attesta comunque su un prevalente piano binario che riguarda l'azione benefica e punitiva. Particolarmente rilevante è infatti la distinzione

\footnotetext{
ne accentuazioni analoghe: «Il fatto che l'ov̌ bili, significa che l'autocomunicazione di Dio non dissipa il mistero, anzi lo rende più profondo; nella rivelazione, però, trapela una traccia di ciò che è Dio. Grazie alla propria ragione, osservando il cosmo, l'uomo può intuire l'esistenza di Dio come $\pi$ ointńs e affermare il legame unidirezionale che unisce la creatura al suo creatore. Rivelandosi, invece, il Signore dischiude all'uomo la sua vicinanza».

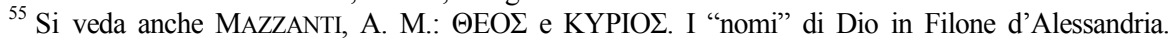
Questioni storico-comparative. Studi Storico Religiosi 5.1 (1981) 15-30.

${ }_{57}^{56}$ FrICK, P.: Divine Providence in Philo of Alexandria. Tübingen 1999, 80.

${ }^{57}$ M. HADAS-LEBEL (Philo of Alexandria. A Thinker in the Jewish Diaspora. Leiden-Boston 2012,

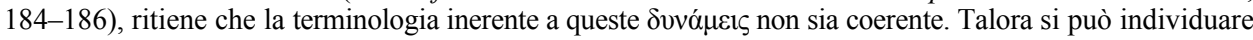
una moltitudine di angeli, in altri passi le connessioni con le Idee platoniche sembrano prevalere. Non bisogna dedurre che le due interpretazioni possano essere assimilate.

${ }_{58}^{58}$ TERMINI (n. 54) 43-44.

${ }^{59}$ FRICK (n. 56) 187-188.
} 
nei termini spesso riscontrabili di $\Theta \varepsilon o ́ \varsigma$ e Kúpı $\varsigma^{60}$ che confluisce infine in un'unica presenza: i poteri punitivi devono essere inclusi nei poteri benefici, domina infatti in assoluto la positività. Filone ne offre due spiegazioni: la legge è adeguata se insieme svolge la funzione di premiare e di punire, il castigo è un monito che spesso comporta il riscatto, il cambiamento secondo una prospettiva non solo personale, ma di una moltitudine. Il riferimento alle vicende argomento della narrazione è indicativa.

E allora il $\gamma$ óp del capitolo 8 è veramente avulso dal ruolo esplicativo di legame fra la trattazione precedente a quella successiva ed è quindi inspiegabile? D.T. Runia, dopo avere fatto alcune annotazioni su possibili nessi nelle argomentazioni scrive: «A contextual reading of the complex passage Legat. 1-7 has proved a useful exercise. Although the passage has few of the formal characteristics of an exordium, its contents are closely related to the subject matter of the entire treatise». ${ }^{61}$ Filone premette ad una narrazione che lo coinvolge emotivamente e che non è aliena da commenti di disprezzo e di rivalsa nel tramandare alla memoria i fautori del dramma perpetrato (74-114; 120; 355), la consapevolezza del significato degli avvenimenti. Quale il nesso fra "storia politica" e "speculazione" filosofica e religiosa? Potremmo parlare di un'interazione o di un'applicazione di contenuti sui dati storici? Non si coglie il presupposto di un'operazione ideologica, ma l'attestazione di un metodo in grado di dare la possibilità di esercizio del giudizio sulle circostanze vissute e quindi sulla storia. I sensi ingannano nella valutazione del presente perché conducono all'opinione che l'elemento dominante della realtà sia il caso, la ragione, quando non è soggetta a corruzione, è invece criterio sicuro per comprendere la presenza essenziale

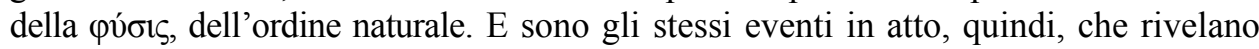
l'esistenza della provvidenza divina nei confronti dell'umanità tutta e in particolare del popolo eletto, quel popolo che è educato a volgersi verso Dio e quindi a vivere nella perfezione morale. Dio e le Potenze sono irraggiungibili nella loro essenza per l'uomo ma le circostanze, pure se drammaticamente avverse, legate cioé a punizioni, rendono evidente alla moltitudine la positività della storia. Di qui prende le mosse la narrazione in cui Filone esprime valutazioni particolari che inevitabilmente rimandano a contenuti già formulati nell'incipit $\mathrm{e}$ a quelle concezioni che sono proprie del suo orizzonte filosofico e religioso.

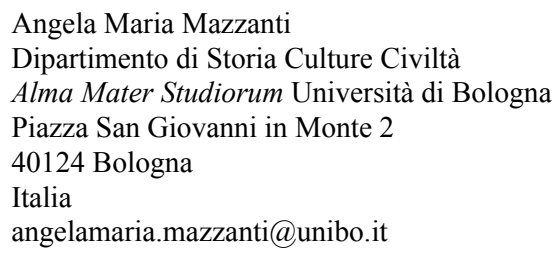

\footnotetext{
${ }^{60}$ Nei Settanta i due termini traducono gli appellativi di Jahweh e di Elohim. In Filone sono citati più volte: si veda, fra l'altro, Abr. 119-132, Mutat. 24.

${ }^{61}$ RUNIA (n. 3) 369.
}

Acta Ant. Hung. 56, 2016 